\title{
Index of Names and Places
}

Note: all references to the body of the text are italicised and are according to the pagination of the editio princeps of Socrates from 1651, marked by square brackets throughout the present edition. All other references are given afterwards in regular Arabic numerals.

Abravanel, Jehuda Leon 516

Acosta, Cristóbal 497

Aegean Sea 212

Aelian 518

Aesop 2, 486, 512

Africa 167, 212-13

Agathon 114, 156, 199, 203-204

Alcibiades 138, 156, 167, 172, 175, 249, 250, 303, 13, 504

Alcmaeon 315

Alexander of Aphrodisia 502

Alexander the Great 488, 509, 516

Al-Ghazālī 510

Alhazen 505, 522

Amon 225

Anacharsis the Scythian 163, 184, 507

Anaxagoras 20-21, 79, 83, 189, 190, 192-94, $197-98,208,211,252,254,11,12,13,491$, 498

Anaxarchus 516

Anaximenes 18, 491

Antaeus 12, 489

Antisthenes 112-13, 201, 303, 11, 502

Anytus 13, 37, 489

Apelles 295, 517

Apis 225

Apollo 3, 5, 59, 8, 486, 492, 495, 513, 518

Aquinas, Thomas 4, 493, 495, 502, 504, 505, 506, 508, 512, 513

Archelaus 90-92, 139, 207-8, 214-16, 12, 498

Archimedes 492, 515

Archytas of Tarentum 84-88, 498

Argus 21, 236, 13, 491, 513

Ariosto, Ludovico 500,507-8, 531

Aristarchus of Samo: 9, 114, 488, 508

Aristarchus of Samothrace 218, 251, 249, 502, 511

Aristippus 44, 167-69, 172, 214, 286, 303, 494, 518

Aristotle $8-9,22,177,214-20,286,451$, $457,11,12,487-88,491-98,500$,
$508-13,516,518-19,521-22,525$, 537

Arrian 509

Asia 154, 212, 244, 283, 287, 495, 509

Athens 187, 234-35, 256, 302, 5, 487, 501, 509, $515,517,540-41$

Atlantic Ocean 70, 284, 510

Attica 154, 287, 489

Aulus Gellius 498, 511-13

Averroes 4, 507

Avicenna 492, 501-2, 510

Bacchus 112

Bacon, Francis 492, 510, 514-15

Bacon, Roger 505

Boccaccio, Giovanni 517

Boccalini, Traiano 514, 516

Bodin, Jean 539

Boethius 211, 142, 504-5, 507, 512

Boiardo, Matteo Maria 487

Botero, Giovanni 514

Bruno, Giordano 491, 493, 498, 504, 507, 513, 534

Byblis $51,121,494$

Calchas 254, 513

Callicles 102-3, 156, 221, 501

Calvin, John 515

Carneades 2, 489, 494

Charmides 113

Chilon 242

Cicero 2, 488-91, 494, 502, 504, 508-9, 513, 516-19

Circe 63, 496

Cleinias 114, 156, 216-17, 246-49, 13, 502

Cohen, Hermann 541

Corinth 234

Cratylus 235-37, 490

Critias 103-5, 203-4, 244-45, 10, 13, 501, 504-5

Crito $64-65,70,96,116,199,10,489,496$, 499,522

Curtius 509

Cyrus 191, 292, 509, 517

Dall'Angelo, Marino 493 
Damon 298, 518

Dante 8, 89, 486-87, 490, 493, 496, 498, 499, $508,510,521,531$

Darius I 511, 516

Delmedigo, Joseph 499, 514, 537

Delphi 8, 286, 492, 513, 518, Dedication, 3, 5, 246

Democritus 19-20, 79, 80-81, 83-84, 147, 175, 209, 230-31, 254, 271, 283, 490-92, 496, 498, 506, 516, 523

De Saavedra Fajardo, Diego 513

Deucalion 277, 515

Diogenes Laertius 487, 494, 497-98, 502-3, 507, 509, 511-12, 517, 525

Diogenes of Sinope 156, 167-69, 230, 286, 304, 489,507

Dion $84,86,88,498$

Dionysodorus 187, 11, 509

Diotima 98-101, 10

Draco 277, 515

Egypt 53, 209, 224-25, 228, 242, 285-86, 12, 495

Elea $18,36,61,235,490,496$

Elean Stranger 11, 496, 61

Empedocles 19, 70, 187-90, 192, 223, 491, 502

Endymion 41, 493

Epictetus 489

Epicurus 493-94, 496, 506, 523

Epirus 244

Erasistratus 506

Erasmus of Rotterdam 495, 508-9, 512, 517, 519

Eridanus 18, 491

Euclid 496-98, 503, 507, 510, 522

Euripus 139, 505

Europe 244

Eusebius of Caesarea 519-20

Euthydemus 198-202, 206-7, 12, 509

Euthyphro 10, 488, 509

Fabrizi dell' Acquapendente, Girolamo 504

Fiamma, Carlo 499

Ficino, Marsilio 505, 508, 515, 518-19, 535, 545

Florus 512

Galen 494, 499-500, 504-6, 517, 524

Galilei, Galileo 488, 497, 499-500, 510, 512, 515

Gilbert, William 497

God 53, 62, 86, 130, 198, 236, 256, 259, 273, 276-77, 288, 291, 307, 313, 490, 514-15, $536,538,540-41$
Gorgias $10,34-38,40,49,56,58,62-63,89$, $117,129,160,193,202,277,9-10,488$, 493, 501-2, 518

Greece $11,154,212,224,244,283,287,307$, 488, 492, 495, 502, 504-5, 507, 511, 513, 516

Helen 122

Heraclitus 18, 59, 60-61, 194, 490

Hercules 9, 112, 314

Hero 53, 495

Herophilus 506

Herostratus 488

Hessus, Helius Eobanus 514

Hippias 4, 242, 244, 256, 272, 277, 280-284, 287, 309, 12-14, 487

Hippocrates 263, 288, 494, 506, 514, 517

Homer 17, 21, 176, 223, 249, 254, 301, 11, 487, 491, 496, 502, 512-14, 518

Horace $289,507,510,514,517-18$

Icarus 280

Indies 212

Ionian Sea 212

Isis 225

Isocrates 300,518

Ixion 509, 194, 229

Italy 84-85, 212, 220, 285

Jupiter, see also Zeus 95, 313, 498, 500, 540

Juvenal 238, 255, 495, 503, 512, 514, 518

Kepler 497, 499-500, 524

Kircher, Athanasius 498, 501

La Boétie, Étienne de 2, 495

La Mothe Le Vayer, François de 532-38

Laches 10, 488

Leucippus 491, 496, 523

Lucan 212, 266, 273, 310, 510, 514-16, 519 , 528

Lucretius 82, 93-94, 211, 257, 308, 492, 494, 496, 498, 499, 503, 506, 510, 514, 519, 523,534

Macedonia 58, 124, 244

Machiavelli 513-14, 516

Maimonides 4, 516

Manfredi, Martino 514

Manilius 108, 501

Manzini, Luigi 486, 493

Medea 109, 501

Mediterranean Sea 212

Medusa 18, 490

Meletus 13, 37, 489

Melissus 17, 43, 60, 194, 231, 488, 490 
Mendelssohn, Moses 7, 530, 541

Milo of Croton 499, 518

Modena, Leon 5, 503

Molière 501

Montaigne, Michel de 2, 10, 488, 490, 492, 494-95, 499, 500, 504, 506-8, 514, 516, $518,532,535,537-38$

Nicias 214, 511

Nile 208-9, 285

Ockham, William of 496, 507, 510

Orpheus 19, 491

Ovid 52-54, 109, 121, 257, 306, 493-95, 498, 502-3, 505, 509, 514-15, 519

Palaemon 217, 511

Paracelsus 517

Parmenides 18, 36, 43, 60, 194, 223, 231, 490, 493, 496

Pausanias 495, 513

Peloponnese 212, 244

Pericles 13, 64, 194-96, 227, 237-42, 249, 251-52, 275, 311

Persia 124, 153, 243-44, 286-87, 488, 509, 511,516

Phaedo 184, 496, 503, 505, 508-9, 518, 530

Phaeton 18, 491

Phoenix 12, 489

Phidias 209, 495

Philolaus 220-24, 12, 511

Piccolomini, Alessandro 499, 506, 516

Plato 4, 21, 61, 113, 152-57, 208, 220-22, 224, $226,228-30,243,246,300,303,315-16$, 11-14, 487-90, 492, 494, 496-98, $501-6,508-11,513,517-18,522-23$, 530,535

Plautus 191, 501, 508-9

Pliny 496-98, 502, 506, 517-18, 528, 537

Plutarch 8, 486-89, 492, 498, 511, 517

Polyphemus 236, 13, 512

Portaleone, Abraham 537

Prodicus 174-76, 11, 508, 518

Prometheus 515

Propertius 499

Protagoras 14, 17, 32, 50, 60-61, 161, 177, 192, 207, 237, 238, 241, 11, 13, 489, 526

Protarchus 127-29, 130, 504

Proteus 135, 191, 248, 280, 504

Provenzali, Moshé 507

Ptolemy 496, 498, 514, 518, 523
Pyrrhus 495

Pythagoras $8,20,189,221,8,487-88,510$, 522

Saliceto, Guglielmo 494

Saturn 95, 138, 11, 495, 500, 504

Sextus Empiricus 1, 2, 6, 487-90, 493-97, 500-501, 503-4, 506, 509, 515, 517-18, $520-21,525-26,528,540$

Scheiner, Christoph 500

Sicily $214,285,488,498,500,511$

Solomon Dedication, 1

Sperelli, Alessandro 499

Strabo 495, 502

Strauss, Leo 530, 541

Suetonius 511, 512

Tacitus 513-14, 516, 518, 541

Tantalus 32, 493

Tasso, Torquato 515, 531

Terence 137, 504, 509

Thaïs 199, 228, 509

Thales $18,71,279,491,497,504,516$

Theaetetus $10,202-4,206,304,12,488-89$, 510

Thebes 129

Themistocles 11,488

Theophrastus 214-20, 12, 496, 501, 511

Thrasymachus $245-46,13,513$

Thucydides 488, 504, 511

Timon 4, 277, 308-9, 315, 14, 487

Tiresias 237, 14

Ulysses 249

Valerius Maximus 488, 516

Verani, Gaetano Felice 512

Vesalius, Andreas 504, 524

Virgil 103, 133-35, 139, 180, 207, 214, 286, 498, $501,504,505,508,510-11,516$

Witelo 497, 506

Xanthippe 148

Xenocrates 221-23, 12, 511

Xenophanes 17, 43, 194, 231, 488, 490, 502

Xenophon 11, 13, 303, 9, 489, 494, 508, 517-18

Xerxes 283, 516

Zeno 43, 235, 493, 511-12

Zeus, see Jupiter 24, 57, 209, 491, 493, 495, 509-10, 515

Zuccolo, Lodovico 512 